\title{
Effects of a magnetic Fe monolayer on the structural and surface electronic properties of $\mathrm{Sb}_{2} \mathrm{Te}_{3}$
}

\author{
Farideh Hajiheidari, ${ }^{1}$ Wei Zhang, ${ }^{1,2}$ and Riccardo Mazzarello ${ }^{1,3, *}$ \\ ${ }^{1}$ Institute for Theoretical Solid State Physics, RWTH Aachen University, D-52074 Aachen, Germany \\ ${ }^{2}$ Center for Advancing Materials Performance from the Nanoscale, State Key Laboratory for Mechanical Behavior of Materials, \\ Xi'an Jiaotong University, Xi'an 710049, People's Republic of China \\ ${ }^{3}$ JARA-FIT and JARA-HPC, RWTH Aachen University, D-52074 Aachen, Germany \\ (Received 22 May 2016; revised manuscript received 26 August 2016; published 15 September 2016)
}

\begin{abstract}
In this paper, we investigate the properties of Fe monolayers deposited on the (111) surface of the topological insulator $\mathrm{Sb}_{2} \mathrm{Te}_{3}$ by density functional theory simulations. We consider high-coverage monolayers and assume ferromagnetic configurations. We show that upon relaxation, the Fe atoms partly penetrate into the surface and that their magnetic moments are reduced due to the chemical interaction with the Te and $\mathrm{Sb}$ atoms of the two topmost layers. We compute the magnetic anisotropy energies and show that the easy axis is in-plane. We investigate hexagonal warping effects and find that the clean $\mathrm{Sb}_{2} \mathrm{Te}_{3}(111)$ displays a large warping term. In spite of this, the surface-state gap for in-plane magnetization is below $1 \mathrm{meV}$. For very high coverages corresponding to three Fe atoms in the unit cell of $\mathrm{Sb}_{2} \mathrm{Te}_{3}(111)$, no surface Dirac cones are observed except in the case of a metastable model consisting of a hexagonal $\mathrm{Fe}$ monolayer lying on top of the substrate, which also exhibits an out-of-plane easy axis. Finally, we discuss the relevance of our paper to recent experiments about magnetic impurities deposited on topological insulators.
\end{abstract}

DOI: 10.1103/PhysRevB.94.125421

\section{INTRODUCTION}

Three-dimensional topological insulators (TIs) possess conducting surface states in the bulk band gap [1-5]. As a result of time-reversal symmetry and strong spin-orbit coupling (SOC), these states exhibit spin-momentum locking $[4,5]$. Furthermore, the surface states are protected against time-reversal-invariant perturbations, such as nonmagnetic impurities. As a result, this type of disorder does not induce localization of the states at the surface, in contrast to standard two-dimensional electron gases.

On the other hand, the presence of magnetic disorder (due, e.g., to magnetic impurities) [6-16] or the proximity to a magnetic system such as a ferromagnetic insulator [17] destroy time-reversal symmetry and can lead to novel phenomena such as the opening of a band gap and an anomalous quantum Hall effect $[4,18]$. These effects have received considerable attention recently [6-21] because of their fundamental interest and their potential applications in spintronics devices integrating TIs with magnetic materials. Reference [8] reported that the deposition of iron atoms on the TI $\mathrm{Bi}_{2} \mathrm{Se}_{3}$ opens a gap at sufficiently high Fe coverages. The claim was based on angle-resolved photoemission spectroscopy (ARPES) experiments. The authors also observed the formation of multiples of Dirac fermions. However, more recent (low-temperature and room-temperature) ARPES measurements did not reveal any gap up to a coverage of 0.3 monolayer (ML) [9]. Furthermore, Honolka et al. [10] performed x-ray magnetic circular dichroism (XMCD) experiments and showed that the easy axis of magnetization of the $\mathrm{Fe}$ adatoms is in-plane, and, for low coverage of $0.01 \mathrm{ML}$, no magnetic ordering occurs (at $T=10 \mathrm{~K}$ ). From these findings, they concluded that such impurities cannot induce a gap. No gap was observed by ARPES for $\mathrm{Fe}$ on the TI $\mathrm{Bi}_{2} \mathrm{Te}_{3}$ at coverage up to one-ML coverage either [11].

\footnotetext{
*mazzarello@physik.rwth-aachen.de
}

More recently, annealing effects were investigated using various experimental tools, as well as ab initio simulations. In a combined scanning tunneling microscopy (STM) and computational study, Schlenk et al. [14] showed that upon thermal annealing at temperatures up to $370 \mathrm{~K}$, the Fe atoms deposited on the $\mathrm{Bi}_{2} \mathrm{Se}_{3}(111)$ surface substitute bismuth in the subsurface layer. Reference [15] focused on the same system and showed that $\mathrm{Fe}$ atoms occupy both interstitial sites and (predominantly) substitutional Bi sites. Furthermore, $\mathrm{X}$-ray absorption spectroscopy experiments indicated that $\mathrm{Fe}$ impurities substitute $\mathrm{Bi}$ atoms within the first quintuple layer already at annealing temperatures of $160 \mathrm{~K}$, whereas hightemperature annealing $(520 \mathrm{~K})$ leads to the formation of the $\alpha$-FeSe phase [16].

The energy-momentum dispersion of the surface states of a TI is usually described by an effective, rotationally invariant two-dimensional Dirac Hamiltonian, $H_{0}=v\left(k_{x} \sigma_{y}-k_{y} \sigma_{x}\right)$, where $\sigma_{x}$ and $\sigma_{y}$ denote Pauli matrices. For such a Hamiltonian, inclusion of a magnetic field $B$, which couples exclusively to the spins, can induce a gap only if it has a nonzero out-of-plane component; however, crystal surfaces lack the $U(1)$ rotational symmetry. As a consequence, higher-order, symmetry-breaking corrections to the Dirac Hamiltonian are needed to describe the properties of the states quantitatively and sometimes even qualitatively. Fu [21] found that for surfaces of TIs with $C_{3 v}$ symmetry, such as the (111) surface of $\mathrm{Bi}_{2} \mathrm{Se}_{3}, \mathrm{Bi}_{2} \mathrm{Te}_{3}$, and $\mathrm{Sb}_{2} \mathrm{Te}_{3}$, the lowest order correction to $H_{0}$ that breaks the $U(1)$ symmetry reads $H_{W}=\lambda\left(k_{+}^{3}+k_{-}^{3}\right) \sigma_{z}$, where $k_{ \pm}=k_{x} \pm i k_{y}$. This term was called a hexagonal warping term. It was obtained by $\mathrm{Fu}$ [21] [together with other corrections that preserve $U(1)$, including a renormalization of the velocity $\left.v_{k} \equiv v\left(1+a k^{2}\right)\right]$ using the $k \cdot p$ expansion near the $\bar{\Gamma}$ point, under the constraint that time-reversal and $C_{3 v}$ symmetries are preserved. Warping terms in hexagonal surfaces had already been considered by Henk et al. [22], who investigated the effects of such terms on the properties of the Rashba-split $L$-surface state of $\mathrm{Au}(111)[23,24]$. Fu [21] also 
showed that in the presence of hexagonal warping terms, an in-plane field $B$ can induce a gap, whose magnitude depends on its direction (being strictly zero only for a discrete set of in-plane directions) and is proportional to $B^{3}$. Thus, it is of great interest to determine whether a ferromagnetic ML deposited on a TI can lead to a measurable gap for in-plane easy axis. Henk et al. [25] showed by density functional theory (DFT) simulations in combination with the coherent potential approximation that for $\mathrm{Mn}$-doped $\mathrm{Bi}_{2} \mathrm{Se}_{3}$ (111) models where the $\mathrm{Mn}$ atoms substitute $\mathrm{Bi}$ atoms in the uppermost layers, a very small gap can be opened or closed for in-plane magnetization as a function of the magnetization's azimuth. In Ref. [25], the $\mathrm{Bi}$ atoms in the topmost layers were replaced by effective $\mathrm{Bi}_{0.9} \mathrm{Mn}_{0.1}$ atoms. Higher magnetic impurity concentrations could be expected to induce larger gaps.

In this paper, the focus is on the (111) surface of $\mathrm{TI} \mathrm{Sb}_{2} \mathrm{Te}_{3}$. Similar to $\mathrm{Bi}_{2} \mathrm{Se}_{3}$, this TI also possesses a simple Dirac cone surface spectrum at the $\bar{\Gamma}$ point of the surface Brillouin zone. The strength of the warping term in $\mathrm{Sb}_{2} \mathrm{Te}_{3}(111)$ is computed and found to be comparable to that of $\mathrm{Bi}_{2} \mathrm{Te}_{3}(111)$. Then, the effects of a ferromagnetic Fe ML deposited on the surface are investigated in the limit of high Fe coverage of one and three MLs. Since our main focus is the understanding of the properties of monolayers obtained by low temperature deposition without annealing, models where the Fe atoms substitute the $\mathrm{Sb}$ cations are not considered; instead, the Fe atoms are placed at different adsorption sites on the surface, and geometry relaxation is performed. The study of high-coverage magnetic MLs is interesting in several respects: Besides inducing strong effective exchange fields, which may have pronounced effects on the TI surface states, they could also lead to the formation of multiple Dirac points, as reported in Ref. [8]. Moreover, it is interesting to determine how the easy axis depends on the coverage and the structure of the Fe ML.

It is found that upon geometry optimization, the Fe atoms penetrate considerably into the substrate, more than for $\mathrm{Bi}_{2} \mathrm{Se}_{3}(111)$ [10], and induce $p$ doping of the surface. For Fe coverage of one ML, the model exhibits a Dirac-like cone at the $\bar{\Gamma}$ point corresponding to surface states hybridized with the Fe $d$ states. Furthermore, additional Fe $d$ bands are present in the bulk band gap of $\mathrm{Sb}_{2} \mathrm{Te}_{3}$. The easy axis turns out to be in-plane and along a direction for which the warping-induced gap should be the largest. In spite of the strong warping term, the surface band gap turns out to be below $1 \mathrm{meV}$. Also shown is the fact that higher coverages (three MLs) strongly modify the structure of the surface by forming a Fe layer in between the uppermost Te and Sb layers. The band structure around the $\bar{\Gamma}$ point is also dramatically changed. In particular, no Dirac cones are observed; instead, two almost dispersionless bands with predominant Fe $d$ character show up. Interestingly, a metastable structure consisting of a densely packed Fe ML lying on top of the surface, which displays an out-of-plane easy axis inducing a large gap between the surface Dirac cones, is also found.

\section{COMPUTATIONAL METHODS}

The simulations are carried out using the plane-wave package Quantum-Espresso [26]. Scalar-relativistic and fully relativistic norm-conserving pseudopotentials [27] and the local density approximation (LDA) are employed [28]. Geometry optimization using scalar-relativistic pseudopotentials (which neglect SOC) is performed; subsequently, we include SOC to investigate the electronic and magnetic properties of the relaxed structures. The magnetic anisotropy energies (MAEs) are determined by computing the total energy for different orientations of the magnetization. Fe coverage of one and three MLs is considered, corresponding to one and three $\mathrm{Fe}$ atoms for each primitive cell of the $\mathrm{Sb}_{2} \mathrm{Te}_{3}(111)$ surface. The $\mathrm{Sb}_{2} \mathrm{Te}_{3}$ substrate is simulated with a slab of 30 layers and a vacuum space of $17 \AA$ between slabs. Thinner slabs would lead to a large spurious gap due to the hybridization between the surface states on opposite surfaces [29]. The Fe ML is put on the top surface of the slab. The Fe atoms and the $\mathrm{Sb}$ and $\mathrm{Te}$ atoms of the four topmost layers of the surface are allowed to relax during structural optimization. $16 \times 16 \times 1$ Monkhorst-Pack meshes [30] are used to perform the integration over the Brillouin zone. The top surfacestate (respectively, bottom surface-state) band structure is determined by calculating the sum of the projections of each Kohn-Sham state onto the atomic orbitals of the $\mathrm{Sb}$ and $\mathrm{Te}$ atoms of the topmost (respectively, bottommost) quintuple layer and by defining as surface states those for which the sum is larger than 0.4. Analogously, the top surface state-Fe bands of the models containing the Fe MLs are determined by including the projections onto the Fe orbitals in the sum and by using the same threshold value. Further computational details are provided in the Supplemental Material [31].

\section{RESULTS}

First, the electronic properties of the clean $\mathrm{Sb}_{2} \mathrm{Te}_{3}(111)$ surface are discussed. The Brillouin zone of the surface is shown in Fig. 1(a). In Figs. 1(b) and 1(c), the band structure of the slab along the $\bar{K}-\bar{\Gamma}-\bar{M}$ direction of the Brillouin zone and the surface state band near the $\bar{\Gamma}$ point are shown, respectively. The Dirac point of the surface states lies at $0.06 \mathrm{eV}$ below the Fermi energy $E_{\mathrm{F}}$. Our results are in agreement with previous work [32]. Experimentally, it is found that $\mathrm{Sb}_{2} \mathrm{Te}_{3}$ typically contains two types of defects, $\mathrm{Sb}$ vacancies and $\mathrm{Sb}_{\mathrm{Te}}$ antisite defects, which lead to $p$ doping of $\mathrm{Sb}_{2} \mathrm{Te}_{3}$ [33]. As a result, the Fermi energy is shifted towards the valence band.

The Hamiltonian up to third order in $\vec{k}$ reads [21]

$$
H=\frac{k^{2}}{2 m^{\prime}}+v_{k}\left(k_{x} \sigma_{y}-k_{y} \sigma_{x}\right)+\lambda\left(k_{+}^{3}+k_{-}^{3}\right) \sigma_{z} .
$$

Its energy spectrum is

$$
E_{ \pm}(k)=\frac{k^{2}}{2 m^{\prime}} \pm \sqrt{v_{k}^{2} k^{2}+\lambda^{2} k^{6} \cos ^{2}(3 \theta)},
$$

where $\theta$ is the azimuth angle of the momentum $\vec{k}$ with respect to $\bar{\Gamma}-\bar{K}$, which, in our models, is parallel to the $x$ axis. Since the correction due to the warping term is zero for $\vec{k}$ along $\bar{\Gamma}-\bar{M}, v$ is determined (from now on, the renormalization of the velocity is neglected) by fitting against the energy dispersion along this direction. The following is obtained: $v=2.47(\mathrm{eV} \AA$ ).

Due to the warping term $H_{W} \equiv \lambda\left(k_{+}^{3}+k_{-}^{3}\right) \sigma_{z}$, the out-ofplane component $S_{z}$ of the spin polarization of the surface 

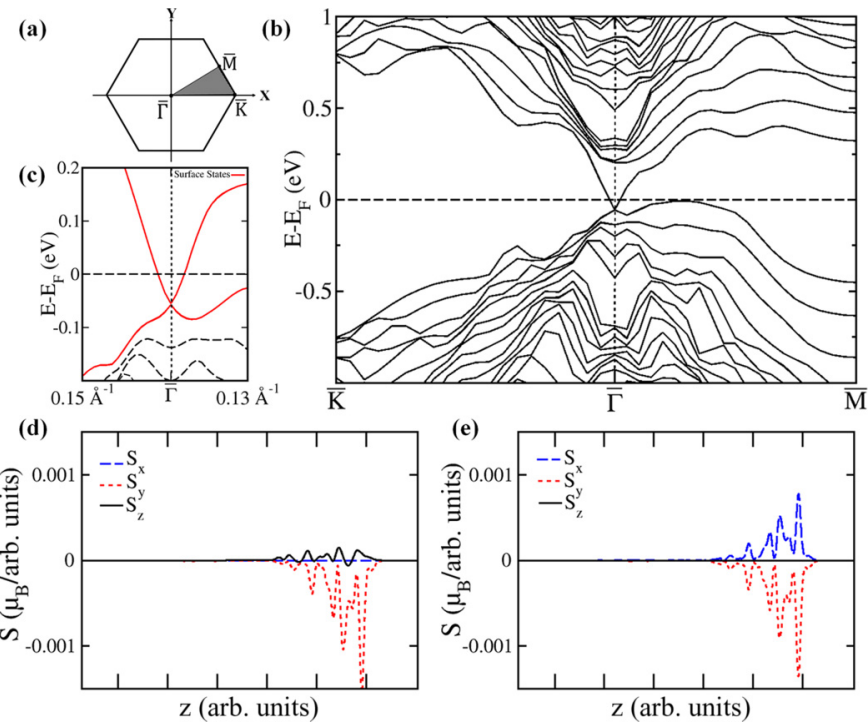

FIG. 1. (a) The surface Brillouin zone of $\mathrm{Sb}_{2} \mathrm{Te}_{3}(111)$. (b) Band structure of $\mathrm{Sb}_{2} \mathrm{Te}_{3}(111)$ along the $\bar{K}-\bar{\Gamma}-\bar{M}$ directions. (c) Zoomed-in view of the surface state (red line) around the $\bar{\Gamma}$ point. (d), (e) Planar averages of the spin polarization density of the surface state at (d) $\vec{k}$ along $\bar{\Gamma}-\bar{K}, \overrightarrow{\mathrm{k}}=(0.009,0.017,0.00) \AA^{-1}$ (with energy $5.5 \mathrm{meV}$ below $E_{\mathrm{F}}$ ), and (e) $\vec{k}$ along $\bar{\Gamma}-\bar{M}, \overrightarrow{\mathrm{k}}=(0.015,0.008,0.00) \AA^{-1}$ (with energy $13.7 \mathrm{meV}$ below $E_{\mathrm{F}}$ ). The tics in the $x$ axis indicate the positions of the six quintuple layers of the $\mathrm{Sb}_{2} \mathrm{Te}_{3}$ slab.

states is generally nonzero. For a given $\vec{k}$, it reads

$$
S_{z}=\cos (3 \theta) / \sqrt{\cos ^{2}(3 \theta)+1 /(k a)^{4}},
$$

where $a \equiv \sqrt{\lambda / v}[21]$. Hence, for a fixed $k, S_{z}$ has a maximum for $\vec{k}$ along $\bar{\Gamma}-\bar{K}$, it is 0 for $\vec{k}$ along $\bar{\Gamma}-\bar{M}$, and it is periodic in $\theta$ with a periodicity of $2 \pi / 3$. In the following, the $z$ axis is assumed to be perpendicular to the $\mathrm{Sb}_{2} \mathrm{Te}_{3}(111)$ surface. Planar averages of the $x, y$, and $z$ component of the spin polarization density of the upper-cone surface states at two different momenta $\vec{k}$, one along the $\bar{\Gamma}-\bar{K}$ direction, $\overrightarrow{\mathrm{k}}=(0.009,0.017,0.00) \AA^{-1}$ (with energy $5.5 \mathrm{meV}$ below $E_{\mathrm{F}}$ ), and a second one along $\bar{\Gamma}-\bar{M}, \overrightarrow{\mathrm{k}}=(0.015,0.008,0.00) \AA^{-1}$ (with energy $13.7 \mathrm{meV}$ below $E_{\mathrm{F}}$ ), are shown in Figs. 1(d) and 1 (e). The $z$ component is large for $\vec{k}$ along $\bar{\Gamma}-\bar{K}$, whereas it is negligible for $\vec{k}$ along $\bar{\Gamma}-\bar{M}$, in agreement with Formula (3) above. From the component $S_{z}$ of the first state, $S_{z}=0.026 \mu_{B}$, $a$, and thus the strength of the warping term $\lambda$ can be estimated: $a=8.2 \AA$ and $\lambda=166.2 \mathrm{eV} \AA^{-3}$ are obtained, to be compared with the values $a=10.9 \AA$ and $\lambda=250 \mathrm{eV}^{-3}$ obtained for $\mathrm{Bi}_{2} \mathrm{Te}_{3}$ by $\mathrm{Fu}$ [21] by fitting the band structure against the experimental data about the Fermi surface of the surface states.

Next, the effects of a Fe ML on the $\mathrm{Sb}_{2} \mathrm{Te}_{3}$ surface are investigated. For one-ML coverage, four different adsorption configurations for the $\mathrm{Fe}$ atoms are considered, namely the two hollow sites with respect to the underlying Te atoms, fcc hollow and hep hollow, the bridge site, and the site on top of a Te atom [see Fig. 2(a)]. Upon relaxation, the Fe atoms penetrate partly into the substrate for all the starting configurations, except for the on top case. Energetically, the most favorable configuration (a)

(b)
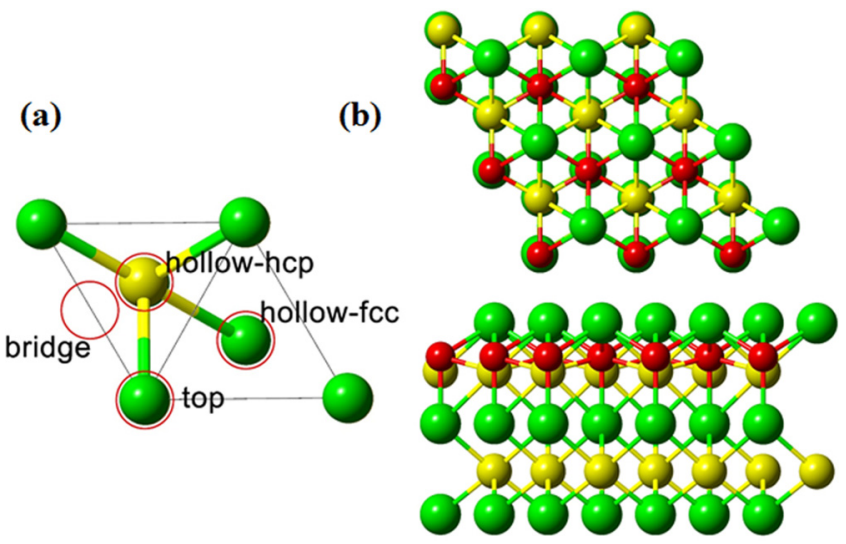

FIG. 2. (a) The four different adsorption configurations for the Fe atoms investigated in this paper are shown. (b) Top and side view of the most stable model of the one-ML Fe on $\mathrm{Sb}_{2} \mathrm{Te}_{3}(111)$. Fe, $\mathrm{Sb}$, and Te atoms are rendered with red, yellow, and green spheres. The most favorable adsorption site for the Fe atoms is the fcc-hollow site. Nevertheless, the Fe atoms partly penetrate into the substrate, as the side view shows.

corresponds to $\mathrm{Fe}$ atoms positioned at fcc-hollow sites but lying in between the topmost Te and Sb layers. The difference between the $z$ coordinate of the $\mathrm{Fe}$ atoms and the Te atoms above is $1.3 \AA$, whereas the distance between the Fe atom and the nearest neighbor Te and $\mathrm{Sb}$ atoms is $2.79 \AA$ and $2.52 \AA$, respectively. Top and side views of the fcc-hollow structure are shown in Fig. 2(b). In the second most stable configuration (with energy $0.70 \mathrm{eV}$ per Fe atom higher than the first one), Fe atoms are at hcp-hollow sites, at slightly lower height, $0.20 \AA$, than the Te atoms. In Ref. [10], it was shown by DFT calculations that in the case of $\mathrm{Bi}_{2} \mathrm{Se}_{3}(111)$ substrates and 0.25-ML Fe coverage, the Fe atoms also sit at fcc-hollow sites but penetrate less deeply into the surface.

The SOC is included, and the MAE is computed for the most stable fcc-hollow configuration. It turns out that the easy axis is in-plane and along the $y$ axis. As shown in Table I, this configuration is $0.8 \mathrm{meV}$ (respectively, $3.0 \mathrm{meV}$ ) per $\mathrm{Fe}$ atom more stable than the magnetization along $x$ (respectively, z). Ab initio calculations showed that the easy axis for $\mathrm{Fe}$ atoms on $\mathrm{Bi}_{2} \mathrm{Se}_{3}$ at fcc-hollow sites is in-plane as well [10]. Moreover, the MAE extracted from XMCD measurements in the latter work (corresponding to low coverages, $0.01 \mathrm{ML}$ ), $1.9 \mathrm{meV}$, is comparable to the one obtained here. Note that the

TABLE I. Energetics and surface electronic properties of a Fe monolayer on $\mathrm{Sb}_{2} \mathrm{Te}_{3}(111)$ at one-ML coverage. The magnetic anisotropy energy $\left(\Delta E_{\mathrm{MAE}}\right)$, the shift of the Dirac point $(\Delta k)$, and the energy gap $\left(E_{\text {gap }}\right)$ of the top surface state for different directions of the Fe magnetization are listed.

\begin{tabular}{lccc}
\hline \hline Magnetization direction & $\Delta E_{\mathrm{MAE}}(\mathrm{meV})$ & $\Delta k\left(\AA^{-1}\right)$ & $E_{\text {gap }}(\mathrm{meV})$ \\
\hline One ML (fcc hollow) & & & \\
$x$ & 0.8 & $8.5 \times 10^{-4}$ & $<0.1$ \\
$y$ & 0.0 & $8.2 \times 10^{-4}$ & 0.4 \\
$z$ & 3.0 & 0.0 & 54 \\
\hline \hline
\end{tabular}


(a)

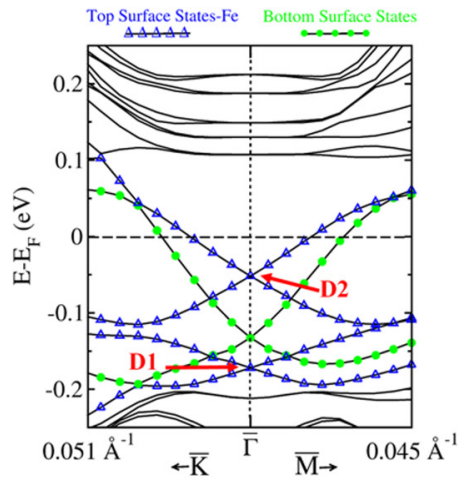

(c)

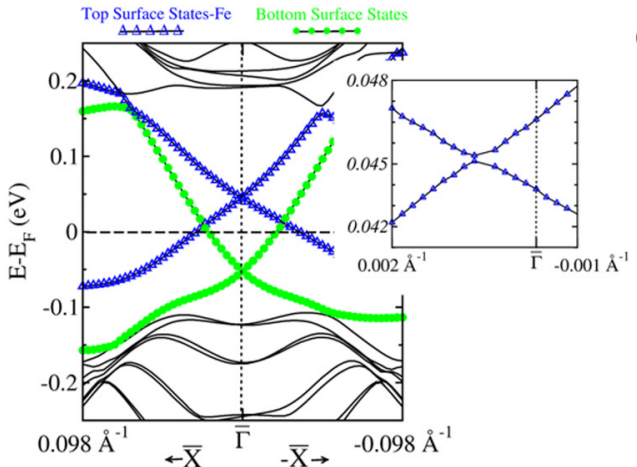

(b)

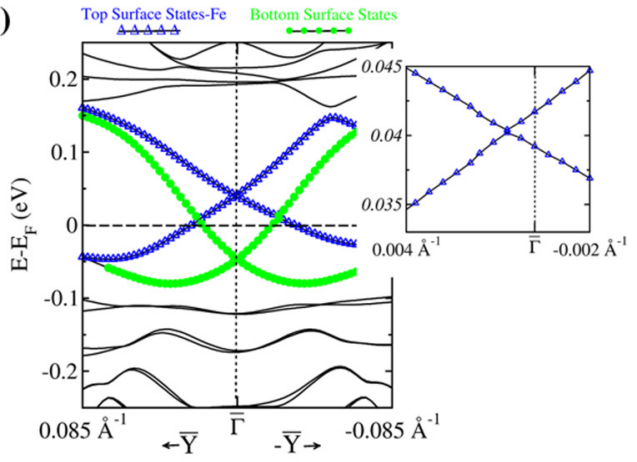

(d)

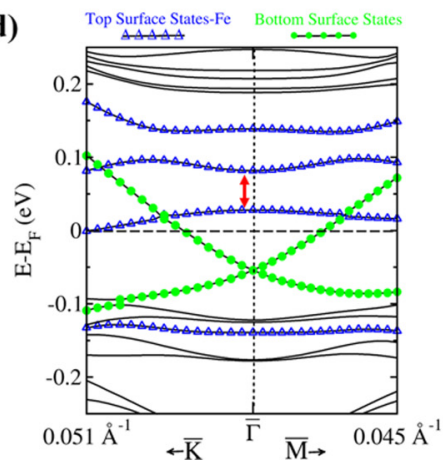

FIG. 3. Band structure of the Fe monolayer on $\mathrm{Sb}_{2} \mathrm{Te}_{3}(111)$ for (a) the nonmagnetic model, (b) the in-plane magnetization along $x$ and (c) along $y$, and (d) out-of-plane magnetization along $z$. Blue and green lines correspond to states localized on the top surface, including the Fe atoms, and on the bottom surface, respectively. No D1 Dirac point is observed near EF for magnetization along $x$ and $y$. The $k$ path for the magnetizations along $x$ and $y$ is perpendicular to the magnetization direction, whereas it is along $\bar{K}-\bar{\Gamma}-\bar{M}$ in (a) and (d). The two insets are zoom-in views near the Dirac point of the top D2 surface state, showing a small shift of the Dirac point and, in (c), a tiny energy gap. The D2 state exhibits a large gap of about $54 \mathrm{meV}$ for out-of-plane magnetization, as shown in (d), where the two relevant bands are indicated with a red arrow. The planar averages of the charge density of the D2 states for the three magnetization directions are shown in the Supplemental Material (see Figs. S3-S5 [31]).

MAEs depend sensitively on the adsorption site. In the case of $\mathrm{Co}$ adatoms on $\mathrm{Bi}_{2} \mathrm{Se}_{3}$, DFT simulations indicate that the stable fcc-hollow site yields an in-plane easy axis [13], whereas the metastable hcp-hollow site [13] and on-top site [12] have out-of-plane axis.

The total magnetization of our model is $1.91 \mu_{B}$, which is significantly smaller than the one reported in Ref. [10] for $\mathrm{Fe}$ on $\mathrm{Bi}_{2} \mathrm{Se}_{3}\left(3.7 \mu_{B}\right)$. This difference stems from the higher coordination of the $\mathrm{Fe}$ atoms on $\mathrm{Sb}_{2} \mathrm{Te}_{3}$ : these atoms form chemical bonds with the three Te atoms above and the three $\mathrm{Sb}$ atoms beneath, which results in strong $p$ - $d$ hybridization, whereas, in the case of $\mathrm{Bi}_{2} \mathrm{Se}_{3}, \mathrm{Fe}$ are bound only to the $\mathrm{Se}$ atoms in the topmost layer. It is stressed, again, that upon annealing, the Fe atoms are expected to substitute cation atoms ( $\mathrm{Sb}$ or $\mathrm{Bi}$ ). The magnetic properties of $3 d$ transition metal atoms at substitutional sites in $\mathrm{Bi}_{2} \mathrm{Se}_{3}, \mathrm{Bi}_{2} \mathrm{Te}_{3}$, and $\mathrm{Sb}_{2} \mathrm{Te}_{3}$ have been thoroughly investigated in Refs. [34] and [35], whereas Refs. [36-40] have focused on substitutional and interstitial $3 d$ magnetic impurities in a closely related group of chalcogenides, the GeSbTe compounds.

The presence of a Fe ML in between the topmost Te and $\mathrm{Sb}$ layers is expected to lead to strong modifications in the band structure. To understand the nature of the relevant states, it is useful to first analyze the band structure obtained after performing a nonmagnetic SOC calculation (without re-optimizing the geometry) [see Fig. 3(a)]. Indeed, new bands with predominant Fe $d$ character appear in the bulk band gap of $\mathrm{Sb}_{2} \mathrm{Te}_{3}$. In particular, there are two Dirac cones at the $\bar{\Gamma}$ point, corresponding to the surface state hybridized with $\mathrm{Fe}$ states (upper point, D2) and to a state mostly localized on the Fe atoms (D1) (see Fig. S1 in the Supplemental Material [31]). More precisely, the D1 state corresponds to a Rashba-split Fe $d$ band and, thus, does not have a topological nature. This is confirmed by the fact that switching off the SOC leads to a spin-degenerate flat Fe $d$ band, as shown in Fig. S2 in the Supplemental Material [31]. The two states exhibit spin-momentum locking, as well as finite $z$ components of the spin along $\bar{\Gamma}-\bar{K}$ due to warping effects, as imposed by the symmetry of the surface. Interestingly, the lower band of the upper energy state D2 bends considerably far from $\bar{\Gamma}$ so as to become nearly parallel to the upper band of the lower energy state D1.

It turns out that switching on of the magnetization [Figs. 3(b)-3(d)] markedly affects the band structure near the Fermi energy. In particular, no D1 state is observed for magnetization along $x$ and $y$. As already stressed, this state has mostly Fe- $d$ character, and, thus, exchange interactions are so strong that they destroy the Dirac nature of the state. 
On the other hand, the main changes in the dispersion of the Dirac-like cone associated with the top surface state D2 (as compared to the bottom surface state, whose dispersion is obviously unaffected by the presence of the Fe ML) can be understood as due to the effective magnetic field originating from the exchange interactions with the Fe electrons, as discussed in the following. Note that the top surface state band D2 does not merge with the bulk states but lies in the bulk gap along the full $\bar{K}-\bar{\Gamma}-\bar{M}$ path, although its orbital character becomes prevalently Fe $d$-like far from the $\bar{\Gamma}$ point. The full band structures and the planar averages of the charge and spin polarization densities of the surface and Fe $d$-like states at selected $k$ points are presented in Figs. S3-S8 in the Supplemental Material [31].

The band dispersion of the top surface state D2 and the size of the gap for Fe spin polarization along different high-symmetry directions is determined: The corresponding plots are shown in Figs. 3(b)-3(d). In the case of magnetization along $x$ and $y$, a $k$ path (around $\bar{\Gamma}$ ) perpendicular to the direction of the polarization is considered, whereas for out-ofplane magnetization, the band structure along a small portion of the $\bar{K}-\bar{\Gamma}-\bar{M}$ path centered at $\bar{\Gamma}$ is shown. If one assumes that the surface states obey the Hamiltonian $H_{0}$, then the presence of a constant in-plane magnetic field $\vec{B}_{\|}$does not induce a gap but only a shift of the Dirac point along the direction perpendicular to the field, $\vec{k}^{*} \equiv g \widehat{z} \times \vec{B}_{||} / v$, where $g$ is the effective electronic $g$ factor. Due to warping effects, however, the magnetic field also opens a gap at the shifted Dirac point, which depends on the direction of the field as follows:

$$
\Delta E=2\left(g B_{\|}\right)^{3} \sin (3 \varphi) \frac{a^{2}}{v^{2}},
$$

where $\varphi$ is the angle between $\vec{B}_{\|}$and $\bar{\Gamma}-\bar{K}$ [21]. If a magnetic film is deposited on a surface of a TI with $C_{3 v}$ symmetry, the exchange interactions induce an effective magnetic field acting on the surface electrons and, thus, may open a gap, even for in-plane magnetization.

When the spin polarization is along $x$, no gap is observed but only a small shift of the Dirac point along the $y$ direction, as shown in the corresponding band structure in Fig. 3(a) (see also Table I). Furthermore, the Fe atoms induce $p$ doping of the surface, as a result of which the cones are shifted to higher energies, slightly above $E_{\mathrm{F}}$, as compared to the Dirac point of the bottom surface. The band structure for Fe magnetization along $y$ is shown in Fig. 3(b). Formula (4) predicts that the gap due to warping effects should reach its maximum value for this magnetization direction. Nevertheless, the gap turns out to be below $1 \mathrm{meV}$. Therefore, the gap is not significantly larger than the one determined in Ref. [25] for Mn-doped $\mathrm{Bi}_{2} \mathrm{Se}_{3}(111)$ models, in spite of the larger coverage. Such small gaps may be detectable only at very low temperatures. A shift of the cones along the $x$ direction is also observed. A magnetization parallel to the $z$ axis instead results in a large gap of about $54 \mathrm{meV}$ [see Fig. 3(d) and Fig. S5 (in the Supplemental Material [31]), which shows the charge-density planar averages of the relevant surface states]. The Fe atoms shift the top surface state band upwards, irrespective of the direction of the magnetization; hence, $\mathrm{Fe}$ atoms act as acceptors. In the case of $\mathrm{Fe}$ on $\mathrm{Bi}_{2} \mathrm{Se}_{3}$, there are conflicting experimental data about the doping effects of Fe atoms $[9,10]$.

The properties of the surface for very high Fe coverages of three MLs are also studied, and the effects on the surface band structure are determined. Different starting adsorption sites for the three $\mathrm{Fe}$ atoms on the $\mathrm{Sb}_{2} \mathrm{Te}_{3}$ primitive cell are considered, and the geometry of the system is fully relaxed. The lowest energy configuration consists of three $\mathrm{Fe}$ atoms sandwiched between the topmost Te and Sb layers (model 1) [see Fig. 4(a)]. The three atoms sit at the hcp-hollow site, fcc-hollow site, and beneath the topmost Te atom, respectively. The resulting structure has the same $C_{3 v}$ symmetry as the clean surface. Due to the intercalated Fe layer, the distance between the $\mathrm{Te}$ and $\mathrm{Sb}$ layer increases considerably. The total magnetization of the system is $5.39 \mu_{B}$. Similar to the one-ML case, the easy axis is in-plane and parallel to the $\bar{\Gamma}-\bar{M}$ direction. The corresponding band structure around the $\bar{\Gamma}$ point is shown in Fig. 4(b), whereas the band structure obtained after performing a nonmagnetic SOC calculation (for the same geometry) is in Fig. 4(c). The latter displays two weakly dispersive bands near $\bar{\Gamma}$, at energies $0.04-0.08 \mathrm{eV}$ above the Fermi level. The two bands are practically doubly degenerate in the momentum range shown and correspond to states mostly localized within the Fe ML. No Dirac-like (top) surface state is found in the bulk band gap. The magnetic band structure in Fig. 4(b) suggests that the in-plane magnetization along $\bar{\Gamma}-\bar{M}$ strongly affects the two bands and lifts the quasidegeneracy. Since this model does not possess Dirac-like surface bands, the properties of the states near the Fermi level are not further discussed here [the analysis is carried out in the Supplemental Material [31] (see Figs. S10-S12)].

Finally, a configuration in which the three Fe atoms form a high-density hexagonal ML lying above the surface [model 2, see Fig. 4(d)] is also considered. In this structure, the nearest-neighbor $\mathrm{Fe}-\mathrm{Fe}$ distance ranges between 2.48 and $2.54 \AA$ (close to the value in the bulk Fe phase), while the average distance between the Fe and Te layer is $2.45 \AA$. The point group symmetry is $C_{3 v}$. It is stressed that this structure corresponds to a local minimum of the potential energy and, thus, does not relax spontaneously into model 1 , although its energy per primitive cell is $3.00 \mathrm{eV}$ higher. It turns out that for model 2, the easy axis is out-of-plane. It is useful to compare nonmagnetic and magnetic band structures for this model as well [Figs. 4(e) and 4(f)]. Interestingly, the nonmagnetic structure also shows two Dirac cones, which correspond to the topological surface state strongly hybridized with the $\mathrm{Fe}$ orbitals [denoted D1 in Fig. 4(f)] and to a Rashba-split state localized mostly on the Fe ML (D2). The nature of the two states has been inferred from the charge density plots shown in Figs. S13 and S14 in the Supplemental Material [31]. Both states exhibit spin-momentum locking. Similar to the one-ML model, the upper band of the lower energy state (state D1) becomes nearly degenerate with the lower band of the upper energy state (state D2) far from $\bar{\Gamma}$. Switching on of the out-of-plane magnetization induces huge gaps at $\bar{\Gamma}$ of the order of $0.8 \mathrm{eV}$ (larger than the bulk band gap) for both states. Therefore, this would be an ideal configuration to investigate magnetic field induced effects on $\mathrm{Sb}_{2} \mathrm{Te}_{3}$. Nevertheless, it is probably very challenging to grow or deposit such metastable structure experimentally, even at very low temperature, due 
(a)

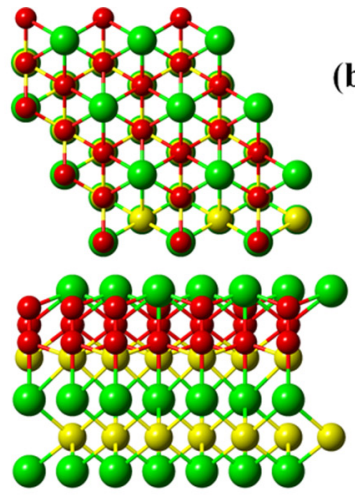

(b)

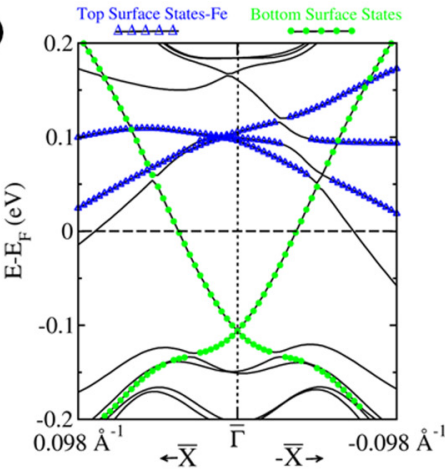

(c)

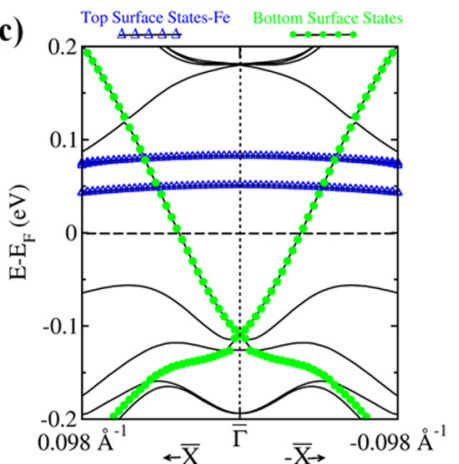

(d)

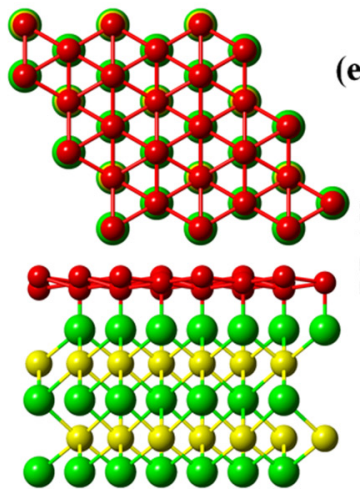

(e)

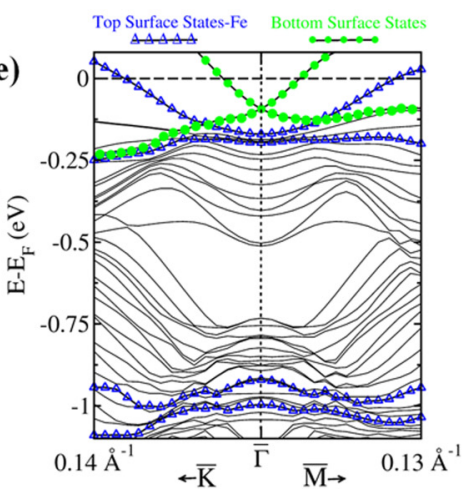

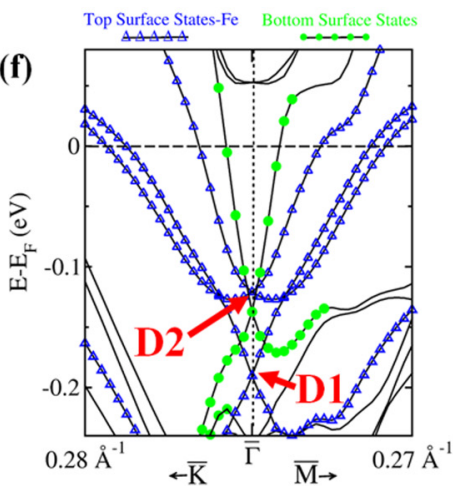

FIG. 4. (a) Top and side view of the most stable model of three-ML Fe on $\mathrm{Sb}_{2} \mathrm{Te}_{3}(111)$ (denoted as model 1 in the main text). (b) Band structure of model 1 for in-plane magnetization along $y$ (easy axis). The $k$ path is perpendicular to the magnetization direction. (c) Nonmagnetic band structure of model 1. (d) Top and side view of a metastable model of the three-ML Fe on $\mathrm{Sb}_{2} \mathrm{Te}_{3}(111)$, wherein the Fe atoms form a layer lying above the $\mathrm{Sb}_{2} \mathrm{Te}_{3}$ surface (model 2). (e) Band structure of model 2 for out-of-plane magnetization along $z$ (easy axis). The $k$ path is along the $\bar{K}-\bar{\Gamma}-\bar{M}$ direction. (f) Nonmagnetic band structure of model 2 . The planar averages of the charge and spin polarization density of the electronic states corresponding to the two Dirac cones are shown in the Supplemental Material (see Figs. S13-S15 [31]) for the nonmagnetic case and for magnetization along $z$.

to the propensity of the $\mathrm{Fe}$ adatoms to partially penetrate into the surface. Further information about the electronic structure of this configuration is provided in the Supplemental Material [31] (see Figs. S13-S17).

\section{DISCUSSION AND CONCLUSIONS}

In this paper, the effects of a Fe ML on the surface states of $\mathrm{Sb}_{2} \mathrm{Te}_{3}(111)$ have been investigated by first-principles simulations. It has been shown that the strength of the warping term in the clean $\mathrm{Sb}_{2} \mathrm{Te}_{3}(111)$ surface is quite large. Then, the structural properties of $\mathrm{Fe}$ adatoms with high coverage (one ML) deposited on this surface have been studied. It is found that (1) the Fe atoms penetrate into the surface and form a layer lying beneath the topmost Te layer, (2) the easy axis of magnetization is in-plane along the $y$ direction, and (3) the surface is $p$ doped. In spite of the large warping effects, the in-plane magnetization does not open a sizable gap in the surface state band structure. Two structures with coverage equal to three MLs have also been considered. In the first stable structure, the Fe atoms are sandwiched between the topmost $\mathrm{Te}$ and $\mathrm{Sb}$ layer, and the easy axis is also along the $y$ direction. In the second metastable structure, the Fe atoms form a ML on top of the uppermost Te layer, and the easy axis is out of plane. As a result, the magnetization induces a very large gap on the surface-state Dirac cone. If realized experimentally, the latter configuration could serve as a model system to study the effects of magnetic perturbations on the surface states of TIs. In this respect, it would be interesting to determine whether magnetic impurities exist that do not penetrate into the $\mathrm{Sb}_{2} \mathrm{Te}_{3}$ (or $\mathrm{Bi}_{2} \mathrm{Se}_{3}$ ) substrate during low-temperature deposition so that they can form stable MLs on top of the surface and possibly exhibit out-of-plane easy axis.

In our paper, ferromagnetic interactions between the Fe atoms have been assumed. In Ref. [41], it was shown that the Ruderman-Kittel-Kasuya-Yosida (RKKY) coupling between two magnetic impurities mediated by the surface state electrons of a TI is ferromagnetic if the Fermi energy is located sufficiently close to the Dirac point so that the Fermi wavelength is larger than the distance between the impurities. However, the actual exchange coupling between $\mathrm{Fe}$ atoms in $\mathrm{Sb}_{2} \mathrm{Te}_{3}$ is more complicated. Recent work based on a first-principles Green's function approach has focused on the exchange parameters between $3 d$ magnetic impurities at substitutional sites in bulk $\mathrm{Bi}_{2} \mathrm{Se}_{3}, \mathrm{Bi}_{2} \mathrm{Te}_{3}$, and $\mathrm{Sb}_{2} \mathrm{Te}_{3}$ [35]. It has been found that the $\mathrm{Fe}$ - and Co-doped bulk phases of the three compounds are antiferromagnetic due to superexchange, whereas doping with $\mathrm{Ti}, \mathrm{V}, \mathrm{Cr}$, and $\mathrm{Mn}$ leads to ferromagnetic configurations stabilized by double exchange and/or free-carrier-mediated indirect exchange [35]. Very recent experiments have indeed con- 
(a)

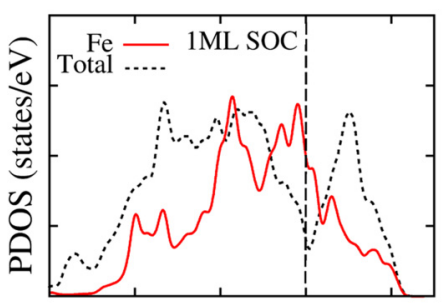

(c)

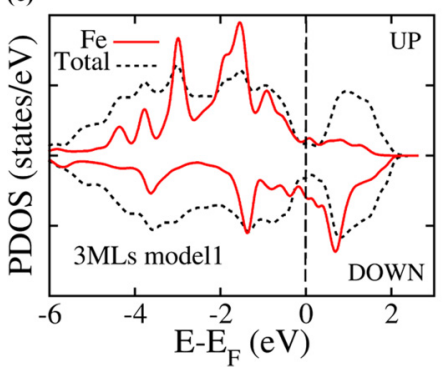

(b)

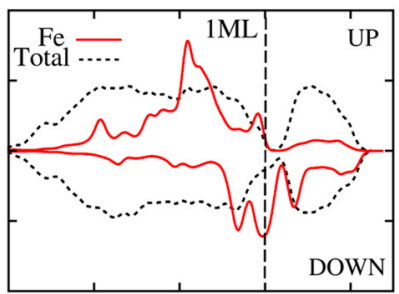

(d)

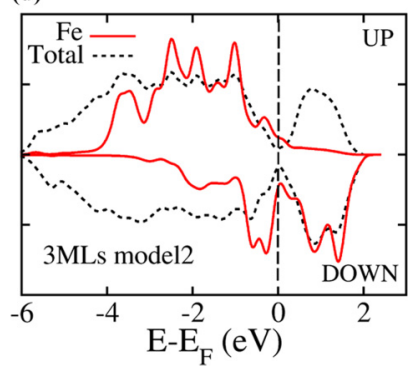

FIG. 5. The total DOS and the PDOS onto the Fe $d$ orbitals for the one Fe ML fcc-hollow model (a) with and (b) without SOC and for the three-ML (c) model 1 and (d) model 2 (both of them without SOC).

firmed that $\mathrm{Cr}$-doped $(\mathrm{Sb}, \mathrm{Bi})_{2} \mathrm{Te}_{3}$ displays carrier-mediated ferromagnetism [42]. In Ref. [39], Fe-doped $\mathrm{Ge}_{2} \mathrm{Sb}_{2} \mathrm{Te}_{5}$ was also found to be antiferromagnetic using a DFT Green's function method. However, for $\mathrm{Fe}$ atoms substituting $\mathrm{Bi}$ in the topmost $\mathrm{Bi}$ layer of the $\mathrm{Bi}_{2} \mathrm{Se}_{3}(111)$ surface, the nearestneighbor exchange coupling constant turns out to be positive (i.e., ferromagnetic), whereas the next- and next-next-nearestneighbor parameters are negative [16]. The three constants are of comparable magnitude, of the order of 1-2 meV. As a result, the $\mathrm{Fe}$ atoms should form a noncollinear magnetic structure [16].

The latter work is the most relevant to our one-ML model, in which, however, $\mathrm{Fe}$ atoms sit at interstitial sites at the very surface instead of substitutional ones. The total density of states (DOS) and the projected DOS (PDOS) onto the Fe $d$ orbitals for this model, obtained including SOC, are shown in Fig. 5(a). The PDOS without SOC are also computed, which enables one to resolve the DOS into majority (assumed to be up) and minority (down) spin contributions [see Fig. 5(b)]. This approximation is justified by the fact that SOC typically affects the strength of the exchange constants weakly. The PDOS indicate that the majority-spin $d$ band is mostly occupied and that there is a large exchange splitting. Further investigations are

required to assess whether antiferromagnetic superexchange prevails over the other exchange mechanisms and whether noncollinear configurations could be stable. In view of the results of Ref. [34], it is expected that interstitial Ti, V, and $\mathrm{Cr}$ one-ML models on $\mathrm{Sb}_{2} \mathrm{Te}_{3}(111)$ should be ferromagnetic. Moreover, they should exhibit similar geometries and SOCinduced anisotropies as the Fe models considered here. The PDOS of the three-ML models are shown in Figs. 5(c) and 5(d). For these systems, itinerant magnetism should also come into play, probably stabilizing the ferromagnetic phase.

In Ref. [10], no experimental evidence for magnetic ordering of $\mathrm{Fe}$ impurities on $\mathrm{Bi}_{2} \mathrm{Se}_{3}$ was found at temperatures down to $T=10 \mathrm{~K}$, in spite of the large anisotropies. This may be due to the low coverages investigated, resulting in very low transition temperatures. In fact, in Ref. [16], the critical temperature $T_{c}$ for the onset of (noncollinear) magnetism in $\mathrm{Bi}_{1.9} \mathrm{Fe}_{0.1} \mathrm{Se}_{3}$ (where $\mathrm{Fe}$ substitutes $\mathrm{Bi}$ only in the subsurface layer) was estimated to be $3.5 \mathrm{~K}$ [16]. $T_{c}$ will obviously increase for increasing $\mathrm{Fe}$ concentration. Similar $T_{c}$ are expected for interstitial Fe MLs with similar Fe concentrations. In Refs. [9] and [11], high coverages were considered; in these works, the absence of a surface-state gap, inferred from the ARPES experiments, may have been due to an in-plane magnetic configuration or a noncollinear structure.

Finally, note that according to Ye et al. [43], the Dzyaloshinskii-Moriya interaction (DMI) $[44,45]$ is of the same magnitude as the conventional RKKY interaction for magnetic impurities at the surface of a TI. The DMI has been shown to yield complex noncollinear configurations in magnetic MLs [46] and magnetic atomic chains [47,48] deposited on metal substrates. For strong TIs with a single Dirac cone such as $\mathrm{Sb}_{2} \mathrm{Te}_{3}$, these interactions could lead to a single-handed spin helix if the Fe impurities are aligned into a chain [43].

\section{ACKNOWLEDGMENTS}

We acknowledge the computational resources provided by JARA-HPC from RWTH Aachen University under Project No. JARA0107. Support from the JARA-FIT Seed Fund programme and from the DFG (German Science Foundation) within the collaborative research centre SFB 917 "Nanoswitches" is also acknowledged. W.Z. gratefully thanks the Young Talent Support Plan of Xi' an Jiaotong University. Finally, we would like to thank the two reviewers for their thoughtful comments, which helped us improve our manuscript significantly.
[1] L. Fu, C. L. Kane, and E. J. Mele, Phys. Rev. Lett. 98, 106803 (2007).

[2] J. E. Moore and L. Balents, Phys. Rev. B 75, 121306 (2007).

[3] R. Roy, Phys. Rev. B 79, 195322 (2009).

[4] M. Z. Hasan and C. L. Kane, Rev. Mod. Phys. 82, 3045 (2010).

[5] X.-L. Qi and S.-C. Zhang, Rev. Mod. Phys. 83, 1057 (2011).
[6] Y. L. Chen, J. H. Chu, J. G. Analytis, Z. K. Liu, K. Igarashi, H. H. Kuo, X. L. Qi, S. K. Mo, R. G. Moore, D. H. Lu, M. Hashimoto, T. Sasagawa, S. C. Zhang, I. R. Fisher, Z. Hussain, and Z. X. Shen, Science 329, 659 (2010).

[7] J. G. Checkelsky, J. T. Ye, Y. Onose, Y. Iwasa, and Y. Tokura, Nat. Phys. 8, 729 (2012). 
[8] L. A. Wray, S. Y. Xu, Y. Q. Xia, D. Hsieh, A. V. Fedorov, Y. S. Hor, R. J. Cava, A. Bansil, H. Lin, and M. Z. Hasan, Nat. Phys. 7, 32 (2011).

[9] M. R. Scholz, J. Sanchez-Barriga, D. Marchenko, A. Varykhalov, A. Volykhov, L. V. Yashina, and O. Rader, Phys. Rev. Lett. 108, 256810 (2012).

[10] J. Honolka, A. A. Khajetoorians, V. Sessi, T. O. Wehling, S. Stepanow, J. L. Mi, B. B. Iversen, T. Schlenk, J. Wiebe, N. B. Brookes, A. I. Lichtenstein, Ph. Hofmann, K. Kern, and R. Wiesendanger, Phys. Rev. Lett. 108, 256811 (2012).

[11] M. R. Scholz, J. Sanchez-Barriga, D. Marchenko, A. Varykhalov, A. Volykhov, L. V. Yashina, and O. Rader, Phys. Status Solidi RRL 7, 139 (2013).

[12] M. Ye, S. V. Eremeev, K. Kuroda, E. E. Krasovskii, E. V. Chulkov, Y. Takeda, Y. Saitoh, K. Okamoto, S. Y. Zhu, K. Miyamoto, M. Arita, M. Nakatake, T. Okuda, Y. Ueda, K. Shimada, H. Namatame, M. Taniguchi, and A. Kimura, Phys. Rev. B 85, 205317 (2012).

[13] T. Eelbo, M. Sikora, G. Bihlmayer, M. Dobrzanski, A. Kozlowski, I. Miotkowski, and R. Wiesendanger, New J. Phys. 15, 113026 (2013).

[14] T. Schlenk, M. Bianchi, M. Koleini, A. Eich, O. Pietzsch, T. O. Wehling, T. Frauenheim, A. Balatsky, J.-L. Mi, B. B. Iversen, J. Wiebe, A. A. Khajetoorians, Ph. Hofmann, and R. Wiesendanger, Phys. Rev. Lett. 110, 126804 (2013).

[15] C.-L. Song, Y.-P. Jiang, Y.-L. Wang, Z. Li, L. Wang, K. He, X. Chen, X.-C. Ma, and Q.-K. Xue, Phys. Rev. B 86, 045441 (2012).

[16] A. Polyakov, H. L. Meyerheim, E. D. Crozier, R. A. Gordon, K. Mohseni, S. Roy, A. Ernst, M. G. Vergniory, X. Zubizarreta, M. M. Otrokov, E. V. Chulkov, and J. Kirschner, Phys. Rev. B 92, 045423 (2015).

[17] F. Katmis, V. Lauter, F. S. Nogueira, B. A. Assaf, M. E. Jamer, P. Wei, B. Satpati, J. W. Freeland, I. Eremin, D. Heiman, P. Jarillo-Herrero, and J. S. Moodera, Nature 533, 513 (2016).

[18] O. A. Pankratov, Phys. Lett. A 121, 360 (1987).

[19] L. Fu and C. L. Kane, Phys. Rev. Lett. 102, 216403 (2009).

[20] A. R. Akhmerov, J. Nilsson, and C. W. J. Beenakker, Phys. Rev. Lett. 102, 216404 (2009).

[21] L. Fu, Phys. Rev. Lett. 103, 266801 (2009).

[22] J. Henk, A. Ernst, and P. Bruno, Phys. Rev. B 68, 165416 (2003).

[23] S. LaShell, B. A. McDougall, and E. Jensen, Phys. Rev. Lett. 77, 3419 (1996).

[24] R. Mazzarello, A. Dal Corso, and E. Tosatti, Surf. Sci. 602, 893 (2008).

[25] J. Henk, M. Flieger, I. V. Maznichenko, I. Mertig, A. Ernst, S. V. Eremeev, and E. V. Chulkov, Phys. Rev. Lett. 109, 076801 (2012).

[26] P. Giannozzi, S. Baroni, N. Bonini, M. Calandra, R. Car, C. Cavazzoni, D. Ceresoli, G. L. Chiarotti, M. Cococcioni, I. Dabo, A. Dal Corso, S. Fabris, G. Fratesi, S. de Gironcoli, R. Gebauer, U. Gerstmann, C. Gougoussis, A. Kokalj, M. Lazzeri, L. Martin-Samos, N. Marzari, F. Mauri, R. Mazzarello, S. Paolini, A. Pasquarello, L. Paulatto, C. Sbraccia, S. Scandolo, G.
Sclauzero, A. P. Seitsonen, A. Smogunov, P. Umari, and R. M. Wentzcovitch, J. Phys.: Condens. Matter 21, 395502 (2009).

[27] N. Troullier and J. L. Martins, Phys. Rev. B 43, 1993 (1991).

[28] J. P. Perdew and A. Zunger, Phys. Rev. B 23, 5048 (1981).

[29] Y. P. Jiang, Y. L. Wang, M. Chen, Z. Li, C. L. Song, K. He, L. L. Wang, X. Chen, X. C. Ma, and Q. K. Xue, Phys. Rev. Lett. 108, 016401 (2012).

[30] H. J. Monkhorst and J. D. Pack, Phys. Rev. B 13, 5188 (1976).

[31] See Supplemental Material at http://link.aps.org/supplemental/ 10.1103/PhysRevB.94.125421 for additional computational details and the analysis of the charge and spin polarization density of the relevant electronic states for the one-ML model and the two three-ML models.

[32] D. Hsieh, Y. Xia, D. Qian, L. Wray, F. Meier, J. H. Dil, J. Osterwalder, L. Patthey, A. V. Fedorov, H. Lin, A. Bansil, D. Grauer, Y. S. Hor, R. J. Cava, and M. Z. Hasan, Phys. Rev. Lett. 103, 146401 (2009).

[33] Y. P. Jiang, Y. Y. Sun, M. Chen, Y. L. Wang, Z. Li, C. L. Song, K. He, L. L. Wang, X. Chen, Q. K. Xue, X. Ma, and S. B. Zhang, Phys. Rev. Lett. 108, 066809 (2012).

[34] P. Larson and W. R. L. Lambrecht, Phys. Rev. B 78, 195207 (2008).

[35] M. G. Vergniory, M. M. Otrokov, D. Thonig, M. Hoffmann, I. V. Maznichenko, M. Geilhufe, X. Zubizarreta, S. Ostanin, A. Marmodoro, J. Henk, W. Hergert, I. Mertig, E. V. Chulkov, and A. Ernst, Phys. Rev. B 89, 165202 (2014).

[36] Y. Li and R. Mazzarello, Adv. Mater. 24, 1429 (2012).

[37] W. Zhang, I. Ronneberger, Y. Li, and R. Mazzarello, Adv. Mater. 24, 4387 (2012).

[38] W. Zhang, I. Ronneberger, Y. Li, and R. Mazzarello, Sci. Adv. Mater. 6, 1655 (2014).

[39] T. Fukushima, H. Katayama-Yoshida, K. Sato, H. Fujii, E. Rabel, R. Zeller, P. H. Dederichs, W. Zhang, and R. Mazzarello, Phys. Rev. B 90, 144417 (2014).

[40] W. Zhang, V. L. Deringer, R. Dronskowski, R. Mazzarello, E. Ma, and M. Wuttig, MRS Bulletin 40, 856 (2015).

[41] Q. Liu, C. X. Liu, C. K. Xu, X. L. Qi, and S. C. Zhang, Phys. Rev. Lett. 102, 156603 (2009).

[42] M. Ye, W. Li, S. Zhu, Y. Takeda, Y. Saitoh, J. Wang, H. Pan, M. Nurmamat, K. Sumida, F. Ji, Z. Liu, H. Yang, Z. Liu, D. Shen, A. Kimura, S. Qiao, and X. Xie, Nat. Comm. 6, 8913 (2015).

[43] F. Ye, G. H. Ding, H. Zhai, and Z. B. Su, Europhys. Lett. 90, 47001 (2010).

[44] I. E. Dzyaloshinskii, Sov. Phys. JETP 5, 1259 (1957).

[45] T. Moriya, Phys. Rev. 120, 91 (1960).

[46] M. Bode, M. Heide, K. von Bergmann, P. Ferriani, S. Heinze, G. Bihlmayer, A. Kubetzka, O. Pietzsch, S. Blügel, and R. Wiesendanger, Nature 447, 190 (2007).

[47] M. Menzel, Y. Mokrousov, R. Wieser, J. E. Bickel, E. Vedmedenko, S. Blügel, S. Heinze, K. von Bergmann, A. Kubetzka, and R. Wiesendanger, Phys. Rev. Lett. 108, 197204 (2012).

[48] R. Mazzarello and E. Tosatti, Phys. Rev. B 79, 134402 (2009). 